\title{
Condições Associadas à Cessação do Uso de Crack entre Usuários em Atendimento
}

\author{
Rogério Lessa Horta ${ }^{1}$ \\ Programa de Pós-Graduação em Saúde Coletiva da Universidade do Vale do Rio dos Sinos, \\ São Leopoldo, RS, Brasil \\ Vanessa Andina Teixeira \\ Programa de Pós-Graduação em Saúde Coletiva da Universidade do Vale do Rio dos Sinos, \\ São Leopoldo, RS, Brasil \\ Larissa Prado Fontoura \\ Fundação Universidade Federal de Ciências da Saúde de Porto Alegre, \\ Porto Alegre, RS, Brasil \\ Simone Poletto \\ Grazieli Oliveira Oliveira \\ Universidade do Vale do Rio dos Sinos, São Leopoldo, RS, Brasil \\ Raquel Oliveira Pinto \\ Secretaria Municipal de Saúde, São Leopoldo, RS, Brasil \\ Alexandre Dido Balbinot \\ Centro Municipal de Atendimento Educacional Especializado Lampadinha, \\ Cachoeirinha, RS, Brasil \\ Luna Strieder Vieira \\ Programa de Pós-Graduação em Epidemiologia da Universidade Federal de Pelotas, \\ Pelotas, RS, Brasil
}

\section{Resumo}

Este artigo apresenta condições associadas à cessação do uso de crack identificadas entre usuários em atendimento. Estudo transversal, com amostragem em bola de neve, reunindo 495 pacientes vinculados a diferentes serviços de saúde da região metropolitana de Porto Alegre (RS) devido ao uso de crack, que responderam a um questionário pré-codificado, padronizado e pré-testado. Os dados foram submetidos à análise multivariada, por regressão de Poisson robusta para estimar a razão de prevalência para o desfecho uso interrompido de crack (referência a um intervalo de 12 semanas ou mais desde o último episódio de uso). As variáveis que aumentaram a probabilidade de abstinência do crack foram: falta de parceiro, ter familiar em tratamento em algum serviço da rede de saúde mental, luto nos últimos 12 meses, reconhecimento de dificuldades sexuais atribuídas ao crack e reconhecimento de comunidades terapêuticas como recurso efetivo. As que diminuíram a probabilidade foram: ter fumado crack com o pai, atender critérios para dependência de crack, auto percepção de saúde ruim e escore positivo para sintomas depressivos no Inventário de Depressão de Beck (BDI). Sem estabelecer nexo causal, o estudo contribui para o avanço do reconhecimento de condições que possam ser integradas ao planejamento terapêutico, com vistas à otimização dos resultados.

Palavras-chave: Crack, usuários de drogas, drogas ilícitas, transtornos relacionados ao uso da cocaína.

Endereço para correspondência: Rua Jari, 671, Apto. 703, Passo D’Areia, Porto Alegre, RS, Brasil 91350-170. E-mail: rogeriohortamed@gmail.com

Este estudo foi financiado pelo Conselho Nacional de Desenvolvimento (CNPq) no Edital 14/2009 - Universal, processo Ministério da Ciência e Tecnologia (MCT)/CNPq 476941/2009-1. 


\title{
Conditions Associated to Cessation of Crack Cocaine Usage among Users in Treatment
}

\begin{abstract}
This paper presents conditions associated with abstinence from crack in a cross-sectional study with a snowball sampling, in a sample of 495 crack users in treatment at different health services in a metropolitan area in southern Brazil. Data were submitted to a multivariate analysis, by robust Poisson regression in order to estimate the prevalence ratio for the outcome abstinence, defined as reference to a period equal or over 12 weeks since the last usage. Remained in association with crack abstinence: lack of partner, relative in mental health treatment, mourning in the last 12 months, sexual difficulties assigned to crack cocaine and knowledge of therapeutic community as effective resource. Appeared on negative association with abstinence: having smocked crack cocaine with the father, attend the criteria for crack cocaine dependence, poor health self perception and BDI score more than minimum. Despite not establishing causation, this paper contributes to the advancement of the recognition of elements that must be integrated into treatment planning, in order to optimize results.
\end{abstract}

Keywords: Crack cocaine, drug users, street drugs, cocaine-related disorders.

\section{Condiciones Asociadas al Cese del Uso de Crack entre Usuarios en Atención}

\section{Resumen}

Este artículo presenta las condiciones asociadas con el absterse del crack. Estudio transversal, con 495 personas en tratamiento debido al uso de crack en diferentes servicios de salud en el sur de Brasil. Los datos se sometieron a análisis multivariante, por regresión de Poisson con ajuste robusto para estimar la razón de prevalencia para el resultado uso interrumpido de crack (referencia a un intervalo de 12 semanas o más desde el último episodio de uso). En asociación con esta variable fueron: la falta de un cónyuge, tener familia en tratamiento en algún servicio de salud mental, luto en los últimos 12 meses, el reconocimiento de dificultades sexuales atribuidos al crack y el reconocimiento de las comunidades terapéuticas como un recurso eficaz. En asociación inversa con el resultado fueron: haber fumado crack con su padre, criterios para dependencia del crack, la auto percepción de su salud como mala y puntuación en el Inventario de Depresión de Beck (BDI) con síntomas leves o superior. Sin establecer la causalidad, el estudio contribuye a la promoción del reconocimiento de las condiciones que se pueden integrar en la planificación de tratamientos, con el fin de optimizar los resultados.

Palabras clave: Cocaína crack, consumidores de drogas, drogas ilícitas, transtornos relacionados con cocaine.

Conhecido nos países desenvolvidos desde meados da década de 80 , o crack é uma droga derivada da cocaína (P. E. M. Ferreira \& Martini, 2001; Karch, 1999). Trata-se de uma substância neuroestimulante, que leva, também por ser fumada, rapidamente a sintomas do tipo euforia, bem-estar e desejo de repetir o uso (Kessler \& Pechansky, 2008). Inúmeros problemas de ordem física, psíquica e social têm sido relacionados ao consumo de crack (P. J. Cunha, Nicastri, Gomes,
Moino, \& Peluso, 2004; O. F. Ferreira, Turchi, Laranjeira, \& Castelo, 2003; Guimarães, Santos, Freitas, \& Araujo, 2008; Kessler \& Pechansky, 2008).

No Brasil, há relatos de uso do crack desde a década de 80 (Duailibi, Ribeiro, \& Laranjeira, 2008), mas seu consumo ganha espaço de forma gradual, ficando mais evidente a partir da virada do milênio, com relatos de uso alguma vez na vida para $0,4 \%$ dos entrevistados, homens e mu- 
lheres, com idades variando de 12 a 65 anos, em 2001, e $0,7 \%$ em 2006, nas 108 maiores cidades do país (Carlini et al., 2006; Carlini, Galduróz, Noto, \& Nappo, 2002; Duailibi et al., 2008). Em Pelotas, contudo, mais recentemente, estudo de base populacional estimou que $2,5 \%$ da amostra com indivíduos com idades entre 18 e 24 anos informaram já ter consumido crack (Narvaez et al., 2014). Já o II Levantamento Nacional de Álcool e Drogas (LENAD II) indica que o uso do crack nos 12 meses anteriores às entrevistas chegou a $2 \%$ no Brasil (Laranjeira et al., 2013).

A dificuldade para chegar à cessação do uso, entre aqueles que passam ao uso continuado, é também um dos problemas que levam pessoas à busca de serviços de saúde. Os sintomas, tanto da intoxicação, quanto da síndrome de abstinência, podem ser tão intensos que os usuários costumam referir grande dificuldade de suportá-los (Bordin, Figlie, \& Laranjeira, 2004). É possível cessar o uso de crack, mas o investimento terapêutico, neste sentido, exige muito em termos de estratégias motivacionais e de prevenção da recaída (Andretta \& Oliveira, 2008; Oliveira \& Nappo, 2008; Orsi \& Oliveira, 2006).

Hoje existem diversas técnicas e recursos terapêuticos propostos para ajudar os usuários de drogas. Os modelos propostos, porém, ainda trabalham com indicadores de adesão e impacto relativamente baixos (Ramos, 2003; V. S. Rodrigues, Horta, Szupszynski, Souza, \& Oliveira, 2013) e há poucos desenvolvidos ou adaptados, especificamente, para a realidade das populações usuárias de crack (V. S. Rodrigues et al., 2013).

No trabalho com substâncias estimulantes, Farabee et al. (2013) identificaram que as estratégias de evitação foram as que geraram a maior parte dos fatores relacionados à permanência sem retorno ao comportamento de consumo. Battjes, Gordon, O'Grady, Kinlock, e Carswell (2003) mapearam a importância da percepção de consequências negativas do uso de drogas entre adolescentes como um importante fator preditivo da adesão a planos de mudança de comportamento envolvendo a cessação do uso. Naquele estudo, o reconhecimento dos danos teve maior peso na adesão ao tratamento que o padrão de uso prévio. Outros estudos que mostrem quais são os fatores importantes para a cessação do uso de crack e mais informações sobre a população usuária de crack são necessárias com vistas a um adequado dimensionamento da rede de atenção à saúde dessas populações.

Este estudo investigou condições que distinguem usuários de crack com uso cessado desta substância daqueles que relataram uso num período ainda considerado recente. Os entrevistados, todos, estavam vinculados a diferentes serviços de saúde na região metropolitana de Porto Alegre (RS), em função do uso dessa substância.

\section{Método}

Trata-se de um estudo transversal com caráter exploratório.

\section{Participantes}

A amostra estudada envolveu 495 indivíduos vinculados a diferentes serviços de saúde da região metropolitana de Porto Alegre (RS). Destes entrevistados, 205 (41,4\%) eram indivíduos que referiram uso de crack há 2 semanas ou menos e 290 (58,6\%) estavam abstinentes, ou seja, sem consumir o crack há 12 semanas ou mais.

No conjunto dos entrevistados houve predomínio de pessoas do sexo masculino $(95,6 \%)$, sem companheira/o $(71,4 \%)$, com idade entre 18 a 30 anos $(66,5 \%)$, com escolaridade de até 8 anos $(60,2 \%)$, com ocupação regular e com renda total da moradia acima de 2 salários mínimos $(59,7 \%)$. Entre os entrevistados, 94,9\% $(n=470)$ preenchiam critérios para dependência de crack. O tempo médio de uso do crack destes indivíduos foi de 6,3 anos $(D P=3,9$ anos $)$, variando de menos de 1 ano até 26 anos. Esta variável teve distribuição normal.

\section{Instrumentos}

Para a coleta de dados, foi utilizado um questionário pré-codificado, padronizado e pré-testado, desenvolvido pela equipe. Algumas variáveis foram pesquisadas pelo emprego de instrumentos específicos, incorporados ao questionário.

A variável dependente foi abstinência de crack. Para a análise os usuários foram divididos em abstinentes e não abstinentes de crack. Como abstinência de crack foi utilizado o ponto de corte de 84 dias ou mais do último episódio de 
consumo avaliado no momento da entrevista. Este intervalo tem sido definido em alguns estudos como o aceitável para medidas de efeito de intervenções e para segurança em termos de reconhecimento da condição abstinente (Ghitza et al., 2010; Gorelick et al., 2005), ainda que represente um intervalo menor que o proposto pelo DSM IV TR (American Psychological Association [APA], 2002). Abstinente, neste sentido, equivale a usuário em condição de uso interrompido, independente da ocorrência ou não de qualquer tipo de manifestação clínica ou mal estar associado à fissura pela substância. Como não abstinentes foi utilizado o ponto de corte de 14 dias ou menos do último episódio de consumo avaliado no momento da entrevista.

Não foram incluídos, nesta análise, entrevistados que informaram último episódio de consumo em período entre duas e doze semanas, para que os grupos se destacassem claramente. Indivíduos sem condições objetivas de responder o questionário no momento da abordagem, por comprometimento de funções neuropsicomotoras, de qualquer etiologia, eram buscados novamente em novas visitas e, persistindo a impossibilidade, manifesta pelo próprio indivíduo, se caracterizava como exclusão. Não foi empregado qualquer instrumento para aferir ou caracterizar a impossibilidade, expressa pela impossibilidade de compreender e responder as questões propostas.

As variáveis independentes foram categorizadas de acordo com a literatura ou de acordo com a distribuição dos entrevistados e organizadas segundo um modelo que propunha que até a ocorrência do desfecho cessação do uso do crack, exista uma cadeia de determinantes hierarquizados onde os níveis acima determinam os níveis abaixo na cadeia. Modelos hierarquizados vem sendo empregados em estudos epidemiológicos, de forma a dar conta de múltiplas relações entre as variáveis, onde as variáveis independentes ora aparecem como fatores de confusão, ora como mediadoras das relações em análise (Albernaz et al., 2003; Vasconcelos, Almeida, \& Nobre, 2001; Victora, Huttly, Fuchs, \& Olinto, 1997). Por este critério, e com base no que a literatura revisada indica como variáveis potencialmente explanatórias para as diferentes condições examinadas neste estudo, os diferentes grupos de variáveis e sua disposição em cada um dos níveis hierárquicos propostos neste modelo são apresentados na Figura 1

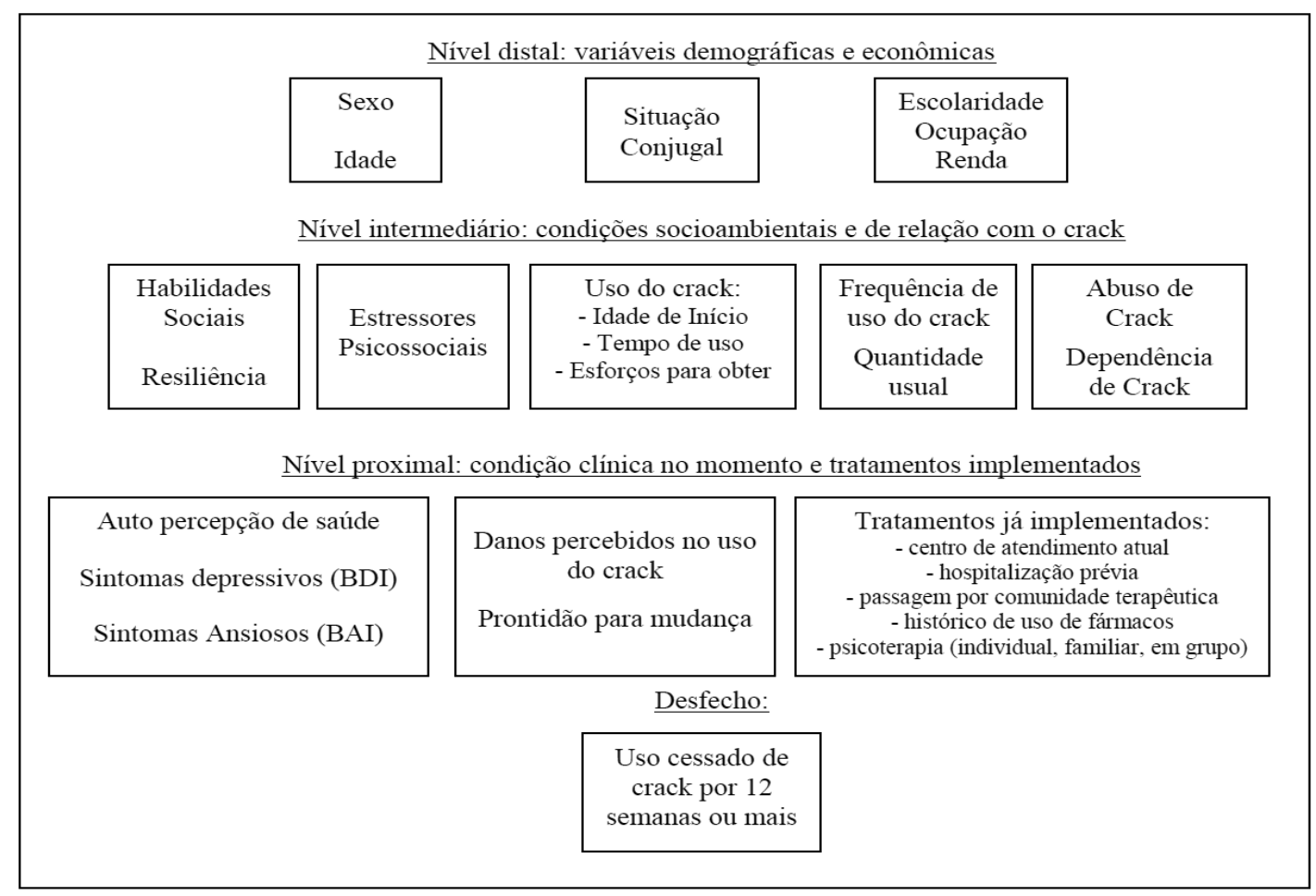

Figura 1. Modelo hierarquizado para análise de condições associadas a uso cessado de crack por 12 semanas ou mais, entre usuários vinculados a diferentes serviços de saúde, Região Metropolitana de Porto Alegre, 2012 ( $n=497)$. BDI = Inventário de Depressão de Beck; BAI = Inventário de Ansiedade de Beck. 
Cada um dos níveis foi composto das seguintes variáveis: (a) Nível distal - variáveis demográficas e econômicas: sexo (masculino/ feminino); idade ( $<18$ anos / 18 anos ou mais); escolaridade (até 8 anos / 9 anos ou mais); situação conjugal (com ou sem companheira ou companheiro); ocupação (sim / não); renda (nenhuma / até 2 salários mínimos / $>2$ salários mínimos); (b) Nível intermediário - condições socioambientais e de relação com o crack. A primeira variável deste grupo é o estágio de desenvolvimento das habilidades sociais, aferido pelo escore no IHS - Inventário de Habilidades Sociais (Del Prette \& Del Prette, 2001). O IHS mostrou, em sua validação correlação alta e significativa $(r=0,90, p=0,001)$ entre duas aplicações e validade concomitante em análise correlacional com a Escala de Assertividade de Rathus $(r=0,79, p=0,01)$. Trata-se de variável discreta, categorizada segundo os pontos de corte padronizados no instrumento. Encontra-se neste grupo, também, a ocorrência de estressores psicossociais. Aqui estão reunidas 20 variáveis dicotômicas, com respostas do tipo sim/não para a pergunta se o entrevistado havia se deparado, nos últimos 12 meses, com alguma das 20 situações indicadas no questionário. Depois aparece a resiliência, avaliada segundo o Inventário de Resiliência (Pesce et al., 2005). Esta é uma variável discreta, categorizada conforme pontos de corte definidos no manual do instrumento. Os esforços empenhados para ter a acesso ao crack reúnem variáveis dicotômicas, com respostas do tipo sim / não para um conjunto de 15 situações usualmente referidas por usuários de drogas como comportamentos que garantem a obtenção da droga. O estudo do histórico e padrão de consumo de crack, que também reuniu variáveis deste nível, incluía informações como idade de início do consumo, tempo de uso do crack, frequência usual de consumo e quantidade consumida em episódios típicos de consumo (variáveis categóricas ou discretas, categorizadas segundo a distribuição dos entrevistados). Dependência de crack se caracterizava por 3 ou mais respostas positivas em 7 propostas e abuso, por 1 ou mais em 4 outros itens propos- tos, coerentes com os critérios do DSM IV TR e como foram empregados pelo estudo National Surveys on Drug Use and Health do Substance Abuse and Mental Health Services Administration (NSDUH; Hughes, Sathe, \& Spagnola, 2009); (c) Nível proximal - condição clínica no momento e tratamentos implementados: estágio de prontidão para a mudança, aferido pelo URICA (University of Rhode Island Change Assessment) validado para o Brasil (Szupszynski \& Oliveira, 2008), com boa consistência interna ( $\alpha$ $=0,657)$, caracterizado como variável discreta, categorizada conforme pontos de corte definidos no manual do instrumento; tratamentos anteriores (variáveis dicotômicas, com respostas do tipo sim/não); auto percepção de saúde (variável categórica, polinomial); níveis de ansiedade e depressão na data da entrevista, aferidos pelo BAI (Inventário de Ansiedade de Beck; consistência interna, $\operatorname{com} \alpha=0,92$, correlação item/total com mediana $=0,60$ e Kappa para aplicações repetidas de 0,75) e BDI (Inventário de Depressão de Beck; que teve na versão em português, avaliação de consistência interna $\operatorname{com} \alpha=0,88$; Beck, Ward, Mendelson, Mock, \& Erbaugh, 1961; J. A. Cunha, 2001), ambas variáveis polinomiais, definidas a partir de pontos de corte para os escores obtidos definidos na validação dos instrumentos); registro de danos percebidos (variáveis dicotômicas, com respostas do tipo sim/não para 20 situações propostas pela equipe de estudo a partir de danos usualmente descritos na literatura revisada); tratamentos implementados, descrito por característica principal do centro de atendimento ao qual estava vinculado (se ambulatorial ou de permanência) - variável polinomial; passagens prévias por hospitais ou comunidades terapêuticas (variáveis categóricas, dicotômicas, com respostas do tipo sim/não); uso de fármacos (variáveis dicotômicas, com respostas do tipo sim/não); histórico de atendimento psicoterápico individual, familiar ou em grupo (variáveis dicotômicas, com respostas do tipo sim/não).

\section{Procedimentos}

Coleta de Dados. A amostragem foi realizada por conveniência, pelo processo bola de neve, 
onde os entrevistados ou os profissionais dos serviços de saúde indicavam outros indivíduos para participarem da pesquisa. Foram visitadas sete clínicas privadas, seis centros de atenção psicossocial (CAPS), um serviço comunitário de atendimento e orientação a usuários e familiares, um hospital, dez comunidades terapêuticas e duas casas de passagem mantidas por comunidades terapêuticas para acolhimento de usuários.

A coleta de dados foi realizada por entrevistadores que foram previamente selecionados e treinados.

Todos os usuários que não tinham condições de responder num primeiro momento foram revisitados e incluídos mais adiante. Foram excluídos por não se encaixarem nos critérios da análise apresentada 22 usuários. Eles não estavam vinculados a serviços de saúde ou tinham feito uso num intervalo entre 2 e 12 semanas.

Os questionários foram codificados e digitados em sistema de dupla entrada no programa Statistical Package for the Social Sciences (SPSS), versão 17.0. Foram checadas 5\% do total das entrevistas para controle de qualidade do preenchimento dos dados.

Análise de Dados. Os dados foram analisados através de estatística descritiva e inferencial. Foram empregados o teste $t$ de Student para comparação entre médias em variáveis com distribuição normal ou o Teste Exato de Fisher para comparação entre médias nas demais variáveis. Comparações entre proporções foram estimadas pelo teste de Qui-quadrado para variáveis categóricas.

Os dados foram submetidos, depois, à análise multivariada por regressão de Poisson, com ajuste robusto da variância, para estimar a correlação entre o desfecho abstinência e as variáveis independentes. Para as razões de prevalência ajustadas, foram incluídas no modelo apenas as variáveis em associação com o desfecho, na análise bruta, em nível de significância de até $95 \%(p<0,05)$. As variáveis eram inseridas no modelo conforme seu nível ou nível superior, de acordo com o modelo hierárquico, permanecendo apenas as variáveis com nível de significância de até 95\% $(p<0,05)$. Não se ve- rificou efeito de delineamento para o desfecho em análise quanto ao local onde foram realizadas as entrevistas.

Este estudo foi aprovado pelo Comitê de Ética em Pesquisa (CEP) da Universidade do Vale do Rio dos Sinos (Unisinos), segundo resolução CEP/Unisinos 027/2010 e foi financiado pelo Conselho Nacional de Desenvolvimento (CNPq) no Edital 14/2009 - Universal, processo Ministério da Ciência e Tecnologia (MCT)/ CNPq 476941/2009-1.

\section{Resultados}

Comparando-se o grupo de entrevistados que se encontravam abstinentes por 12 semanas ou mais com aqueles que relataram uso da droga há 2 semanas ou menos, não foram encontradas diferenças significativas quanto a sexo ( $p$ $=0,694)$, idade $(p=0,227)$, escolaridade $(p=$ $0,074)$, ocupação $(p=0,211)$, idade de início no consumo do crack $(p=0,060)$, tempo de uso do crack $(p=0,700)$, tempo decorrido desde a primeira busca por atendimento em função do uso de crack $(p=0,417)$, número de hospitalizações $(p=0,298)$, nem quanto ao número de critérios positivos para dependência do crack ( $p$ $=0,115)$.

A Tabela 1 mostra as variáveis associadas com o desfecho na análise bruta e seu comportamento na análise ajustada. Permaneceram associadas ao desfecho as seguintes condições: não ter companheiro/a, ter familiar em atendimento em algum serviço da rede de atenção à saúde mental, ter experimentado luto de familiar ou amigo nos 12 meses anteriores às entrevistas, perceber dificuldades sexuais atribuídas, pelo entrevistado, ao consumo de crack e reconhecer comunidades terapêuticas como ajuda para promoção de abstinência. Em associação inversa ao desfecho proposto apareceram as seguintes condições: ter fumado crack com o pai alguma vez na vida, preencher critérios para dependência do crack, perceber a própria saúde como muito ruim e apresentar escore no BDI indicando sintomas depressivos, já a partir do nível leve (12 a 19 pontos em 63). 
Tabela 1

Razões de Prevalência (RP) e Respectivos Intervalos de Confiança (IC 95\%) para Abstinência de Crack em Indivíduos em Tratamento, Região Metropolitana de Porto Alegre - RS, 2012 (n = 495)

\begin{tabular}{|c|c|c|c|c|}
\hline \multirow{2}{*}{ Variável } & \multicolumn{2}{|c|}{ Análise bruta } & \multicolumn{2}{|c|}{ Análise ajustada ${ }^{a}$} \\
\hline & RP (IC 95\%) & $p$ valor & RP (IC 95\%) & $p$ valor \\
\hline Situação conjugal & & $0,004 * *$ & & $0,004 * *$ \\
\hline Com companheiro/a & Referência & & Referência & \\
\hline Sem companheiro/a & $1,11(1,04-1,19)$ & & $1,11(1,04-1,19)$ & \\
\hline $\begin{array}{l}\text { Familiar em atendimento em saúde mental } \\
\text { Não } \\
\text { Sim }\end{array}$ & $\begin{array}{c}\text { Referência } \\
1,11(1,04-1,18)\end{array}$ & $0,001 * *$ & $\begin{array}{c}\text { Referência } \\
1,10(1,03-1,17)\end{array}$ & $0,004 * *$ \\
\hline $\begin{array}{l}\text { Idade que usou crack pela primeira vez } \\
\text { Até os } 18 \text { anos } \\
\text { Acima dos } 18 \text { anos }\end{array}$ & $\begin{array}{c}\text { Referência } \\
1,08(1,02-1,14)\end{array}$ & $0,006^{* *}$ & $\begin{array}{c}\text { Referência } \\
1,08(0,99-1,17)\end{array}$ & $0,080 * *$ \\
\hline $\begin{array}{l}\text { Já ter fumado crack com o pai } \\
\text { Não } \\
\text { Sim }\end{array}$ & $\begin{array}{c}\text { Referência } \\
0,89(0,80-0,99)\end{array}$ & $0,028 * *$ & $\begin{array}{c}\text { Referência } \\
0,83(0,73-0,95)\end{array}$ & $0,009 * *$ \\
\hline $\begin{array}{l}\text { Dependência de crack } \\
\text { Não } \\
\text { Sim }\end{array}$ & $\begin{array}{c}\text { Referência } \\
0,84(0,78-0,90)\end{array}$ & $0,001 * *$ & $\begin{array}{c}\text { Referência } \\
0,91(0,83-0,99)\end{array}$ & $0,038 * *$ \\
\hline $\begin{array}{l}\text { Luto nos últimos } 12 \text { meses } \\
\text { Não } \\
\text { Sim }\end{array}$ & $\begin{array}{c}\text { Referência } \\
1,09(1,02-1,15)\end{array}$ & $0,005 * *$ & $\begin{array}{c}\text { Referência } \\
1,07(1,02-1,13)\end{array}$ & $0,007 * *$ \\
\hline $\begin{array}{l}\text { Brigas em casa atribuídas ao crack } \\
\text { Não } \\
\text { Sim }\end{array}$ & $\begin{array}{c}\text { Referência } \\
0,91(0,85-0,98)\end{array}$ & $0,015^{* *}$ & $\begin{array}{c}\text { Referência } \\
0,95(0,88-1,03)\end{array}$ & $0,239 * *$ \\
\hline $\begin{array}{l}\text { Prisão atribuída ao crack } \\
\text { Não } \\
\text { Sim }\end{array}$ & $\begin{array}{c}\text { Referência } \\
0,93(0,88-0,99)\end{array}$ & $0,034 * *$ & $\begin{array}{c}\text { Referência } \\
0,99(0,92-1,04)\end{array}$ & $0,471 * *$ \\
\hline $\begin{array}{l}\text { Dificuldades sexuais atribuídas ao crack } \\
\text { Não } \\
\text { Sim }\end{array}$ & $\begin{array}{c}\text { Referência } \\
1,07(1,01-1,13)\end{array}$ & $0,015^{* *}$ & $\begin{array}{c}\text { Referência } \\
1,08(1,03-1,13)\end{array}$ & $0,003 * *$ \\
\hline $\begin{array}{l}\text { Perda da moradia atribuída ao crack } \\
\text { Não } \\
\text { Sim }\end{array}$ & $\begin{array}{c}\text { Referência } \\
0,94(0,89-0,99)\end{array}$ & $0,023 * *$ & $\begin{array}{c}\text { Referência } \\
0,98(0,93-1,03)\end{array}$ & $0,351 * *$ \\
\hline $\begin{array}{l}\text { Acreditar que o emprego que não aceita } \\
\text { o consumo de crack ajuda a deixar de usar } \\
\text { Não } \\
\text { Sim }\end{array}$ & $\begin{array}{c}\text { Referência } \\
0,92(0,87-0,98)\end{array}$ & $0,006 * *$ & $\begin{array}{c}\text { Referência } \\
0,96(0,91-1,01)\end{array}$ & $0,086^{* *}$ \\
\hline $\begin{array}{l}\text { Comunidade terapêutica ajuda a deixar de usar } \\
\text { Não } \\
\text { Sim }\end{array}$ & $\begin{array}{c}\text { Referência } \\
1,15(1,06-1,25)\end{array}$ & $0,001 * *$ & $\begin{array}{c}\text { Referência } \\
1,10(1,02-1,18)\end{array}$ & $0,016^{* *}$ \\
\hline $\begin{array}{l}\text { Já dormiu na rua para conseguir fumar crack } \\
\text { Não } \\
\text { Sim }\end{array}$ & $\begin{array}{c}\text { Referência } \\
0,92(0,87-0,97)\end{array}$ & $0,003 * *$ & $\begin{array}{c}\text { Referência } \\
0,97(0,92-1,02)\end{array}$ & $0,214 * *$ \\
\hline $\begin{array}{l}\text { Usa bebidas alcoólicas e quer parar } \\
\text { Não } \\
\text { Sim }\end{array}$ & $\begin{array}{c}\text { Referência } \\
1,10(1,02-1,18)\end{array}$ & $0,009 * *$ & $\begin{array}{c}\text { Referência } \\
1,15(0,94-1,43)\end{array}$ & $0,168 * *$ \\
\hline
\end{tabular}


(continuação)

\begin{tabular}{|c|c|c|c|c|}
\hline \multirow{2}{*}{ Variável } & \multicolumn{2}{|c|}{ Análise bruta } & \multicolumn{2}{|c|}{ Análise ajustada ${ }^{a}$} \\
\hline & RP (IC 95\%) & $p$ valor & RP (IC 95\%) & $p$ valor \\
\hline Usa maconha e quer parar & & $0,011 * *$ & & $0,323 * *$ \\
\hline Não & Referência & & Referência & \\
\hline Sim & $1,11(1,02-1,21)$ & & $1,10(0,91-1,33)$ & \\
\hline Autopercepção de saúde & & $0,001 * *$ & & $0,001 *$ \\
\hline Muito boa & Referência & & Referência & \\
\hline Boa & $0,88(0,84-0,93)$ & & $0,92(0,87-0,98)$ & \\
\hline Ruim & $0,74(0,67-0,82)$ & & $0,81(0,73-0,90)$ & \\
\hline Muito ruim & $0,56(0,53-0,58)$ & & $0,68(0,61-0,76)$ & \\
\hline BDI & & $0,001^{*}$ & & $0,002 *$ \\
\hline Mínimo & Referência & & 1 & \\
\hline Leve & $0,88(0,83-0,94)$ & & $0,93(0,87-0,98)$ & \\
\hline Moderado & $0,80(0,74-0,86)$ & & $0,89(0,82-0,96)$ & \\
\hline Grave & $0,76(0,64-0,89)$ & & $0,92(0,78-1,10)$ & \\
\hline BAI & & $0,001^{*}$ & & $0,413^{*}$ \\
\hline Mínimo & Referência & & Referência & \\
\hline Leve & $0,92(0,86-0,98)$ & & $0,97(0,90-1,04)$ & \\
\hline Moderado & $0,88(0,81-0,95)$ & & $0,98(0,91-1,06)$ & \\
\hline Grave & $0,76(0,67-0,85)$ & & $0,94(0,81-1,08)$ & \\
\hline
\end{tabular}

Notas. BDI = Inventário de Depressão de Beck; BAI = Inventário de Ansiedade de Beck. *Teste Wald p/ tendência linear e **heterogeneidade de proporções.

a Análise ajustada com variáveis com $p<0,05$.

\section{Discussão}

Este estudo discrimina algumas condições relatadas por usuários de crack como fatores que contribuem aumentando a probabilidade deles estarem com uso cessado de crack por 12 semanas ou mais e, também, destaca outras condições se associam, reduzindo a probabilidade de estar sem usar a droga naquele intervalo de tempo.

Diferente do que se observa na literatura para outras drogas, não ter companheira apareceu como facilitador da abstinência (Mehta et al., 2012). Tal resultado pode ser explicado pelo fato de integrantes familiares serem, eventualmente, parceiros de uso de crack e não apenas vítimas da desordem familiar decorrente do crack. Entretanto, quando há algum familiar em atendimento em serviços de saúde mental a probabilidade de cessar o uso de crack é maior. Este dado parece indicar que o engajamento dos familiares, não necessariamente cônjuges, nas iniciativas de busca de tratamento, apoio e mudança de comportamento qualificam a resposta aos tratamentos propostos. Este efeito pode ter múltiplas explicações, não verificadas no questionário empregado: outros familiares podem estar em atendimento por também apresentarem problemas com drogas e o grupo estar em um investimento compartilhado, com apoio mútuo; familiares em tratamento contribuem para o padrão de conflitos familiares ser atenuado e isso contribui para a promoção da abstinência; ou um familiar em contato com o serviço de saúde facilita o acesso do usuário aos centros de atendimento, aumentando as chances de se estabelecerem vínculo e resposta ao tratamento (Guimarães et al., 2008; Horta, Horta, \& Pinheiro, 2006; Pratta \& Santos, 2006; Schenker \& Minayo, 2005).

Ainda na esfera familiar, mas com efeito prejudicial, destaca-se o uso do crack compartilhado com o pai. Este resultado reforça ainda mais a indicação de inclusão de dados familiares em estudos com usuários de drogas. Este dado não está muito evidente em outros estudos, mas indica que a família pode ser tanto um suporte 
quanto um obstáculo à mudança de comportamento (Horta et al., 2006).

No que tange a percepção dos entrevistados em relação às comunidades terapêuticas, observou-se que aqueles que as reconheceram como efetivas na interrupção do consumo do crack tiveram maior probabilidade de cessar o uso de crack do que aqueles que não reconheceram. Isto contribui para a impressão de que o vínculo com os centros de atendimento aumenta a chance de um usuário de crack poder sustentar a interrupção do uso. O mesmo não se repetiu para a hospitalização, CAPS ou para outros serviços. Apesar de a análise multivariada ter incluído todas as variáveis disponíveis, contemplando as passagens por diferentes serviços, o tipo de serviço de inserção mais recente e todos os entrevistados estarem em atendimento, o achado pode refletir apenas uma opinião do momento das entrevistas e não deve ser assumida como indicativo de avaliação de efetividade comparada dos serviços. Mas serve como indicativa da necessidade que usuários de crack costumam manifestar nos atendimentos de um suporte clínico com cuidado mais próximo, atendimentos frequentes e promoção mais intensa da sensação de proteção em relação à fissura e à possibilidade de reiniciar o consumo.

Atendimentos em regime interno, com possibilidade de longa permanência podem acenar com mais deste componente, percebido como apoio. Comunidade terapêutica pode estar representando uma modalidade de tratamento, mas é um dispositivo com forte componente de inserção em grupo social em ambiente alternativo. Qualquer análise destes dados deve considerar isso. Estudos de cunho qualitativo poderão representar oportunidade de compreensão mais profunda da informação aqui acessada e da importância dos diferentes vetores de apoio, em grupos sociais, comunitários ou familiares.

Em relação a algumas exposições que diminuem a qualidade de vida, podemos citar primeiramente os sintomas depressivos e a dependência de crack. Estudos mostram que usuários que se mantêm abstinentes por mais tempo têm menos sintomas depressivos, além de preencherem um menor número de critérios entre os propostos no DSM IV TR (APA, 2002) para dependência
(Duailibi et al., 2008; Guimarães et al., 2008; Horta, Horta, Rosset, \& Horta, 2011). Estes achados reforçam a importância de uma ampla investigação clínica quando usuários de crack ingressam em atendimento, com mapeamento de critérios para diagnóstico de dependência e sintomas depressivos, buscando a integralidade dos cuidados como diretriz, por mais especializado que este seja.

Neste estudo, todos os vinte danos apresentados no questionário tiveram ocorrência elevada de respostas positivas, mas apenas em relação à queixa de dificuldades sexuais atribuídas pelo entrevistado ao uso do crack foi detectada diferença significativa $(p=0,003)$ entre usuários em uso ativo e abstinentes. Mesmo a magnitude da diferença encontrada não sendo grande, é plausível imaginar que a ocorrência de disfunções sexuais contribua para a ampliação de sua motivação para a mudança. $O$ fato de dificuldades sexuais terem sido mais reconhecidas como danos por usuários em abstinência pode também ter sido devido ao acaso e será oportuno aguardar dados de outros estudos para que se amplie a avaliação desta relação.

É difícil estabelecer, por exemplo, com base no instrumento empregado e no delineamento deste estudo, se as dificuldades sexuais motivaram a abstinência ou o contrário. $\mathrm{O}$ uso de drogas, particularmente o crack, tem sido descrito como um facilitador na tarefa de buscar relacionamentos e atividade sexual, inclusive na forma de prostituição, promiscuidade ou atividade sexual sem proteção (Oliveira \& Nappo, 2008). No entanto, a pergunta no questionário solicitava que o usuário indicasse se percebia disfunção sexual como algo decorrente do uso do crack, então a resposta positiva sugere que desordens da atividade sexual tenham antecedido a mudança de comportamento, chegando à cessação de consumo.

Como último preditor de abstinência de crack ressalta-se a ocorrência de luto nos 12 meses que antecederam a entrevista. Usuários em luto apresentaram maior probabilidade de se abster da droga, reafirmando as indicações de que alguns estressores podem ter o papel de gatilhos para a mudança de comportamento em relação 
às substâncias psicoativas (Andretta \& Oliveira, 2008; Bordin et al., 2004; Cahill, Adinoff, Hosig, Muller, \& Pulliam, 2003). Chama atenção o fato de que a condição de luto usualmente inclui sintomas depressivos, mas o registro de luto e a evidência de sintomas depressivos tiveram comportamentos inversos. Isso pode indicar que as associações encontradas, todas de baixa magnitude, se devem ao acaso, mas pode sugerir também que a vivência de luto contribua na tomada de decisão para a mudança de comportamento, que dependerá, entre outras coisas, da superação dos sintomas depressivos. Tal construção parece plausível uma vez que a vivência de luto poderia ter ocorrido até 12 meses antes das entrevistas e a evidência de sintomas depressivos era daquele momento, dizia respeito à condição do usuário durante a entrevista.

Todos estes achados merecem aprofundamento com estudos complementares, especialmente, com delineamento qualitativo. Há relativamente poucas pesquisas sobre as condições associadas à cessação de consumo de drogas, em especial o crack (V. S. Rodrigues et al., 2013), prevalecendo as pesquisas sobre os fatores protetores ou de risco em relação à iniciação do uso (Chen \& Kandel, 1998; García de Jesús \& Ferriani, 2008; Horta et al., 2006; Schenker \& Minayo, 2005).

Como pontos positivos podemos citar a produção de registros da existência de indivíduos que cessam o uso do crack e o estimulo da compreensão dos fatores pelos quais esta mudança de comportamento pode ser viabilizada. No entanto, permanecem outras lacunas, como a necessidade de mensurações longitudinais que clarifiquem a relação entre um episódio de uso de drogas e episódios de transtornos mentais, já apontada há algum tempo (Lopes \& Coutinho, 1999).

Como limitações devemos apontar a amostragem em bola de neve, geradora de viés de seleção, o uso de amostragem não probabilística expõe o estudo à possibilidade de inclusão de indivíduos mais dispostos a participarem da pesquisa, além de não formarem amostras representativas (Lopes \& Coutinho, 1999; Sanchez, Oliveira, Ribeiro, \& Nappo, 2010). No entanto, estudos que adotam tais amostras são úteis para verificar se um fenômeno ocorre no universo em que estão inseridos, os resultados registram sua ocorrência e dão ideia da sua magnitude e importância, fornecendo base para o trabalho futuros (O. F. Ferreira et al., 2003). A justificativa para o uso deste tipo de amostra são as baixas prevalências do consumo de crack somadas a possibilidade dos usuários não serem encontrados em casa ou terem deixado o domicílio, o que praticamente inviabilizaria uma logística para uma coleta de dados em amostra probabilística em estudo de base populacional.

Outra limitação é o uso de um instrumento do tipo recordatório que foi elaborado pelos autores para a investigação dos padrões de uso de substância e da ocorrência de estressores psicossociais, que não garante a validade e fidedignidade dos dados gerados pela entrevista, podendo levar a viés de informação. Tal viés é inerente às investigações com recordatório e com dados referidos, que dependem da capacidade de memorização dos entrevistados ou de outros atravessamentos de ordem cognitiva ou subjetiva (M. A. P. Rodrigues, Facchini, \& Lima, 2006). Há ampliação deste risco em estudos com usuários de crack, que têm potencial para comprometimento das funções cognitivas (P. J. Cunha et al., 2004) e, neste caso, também por estarem em atendimento nos serviços visitados. Para minimização destes riscos, foram tomadas providências no sentido de enfatizar, na formulação das questões, o período de tempo a que cada variável correspondia, com redação clara e com treinamento do grupo de entrevistadores reforçando a necessidade de indicar o período com destaque, dando tempo aos entrevistados para que buscassem as informações. As entrevistas eram individuais, realizadas com garantias de confidencialidade, sempre enfatizando que não haveria qualquer distinção quanto às respostas fornecidas e que a equipe de entrevistadores não atuava nos serviços e não tinha qualquer outra inserção ou vínculo com as equipes, exceto pelo estudo em si. Além disso, a condição abstinente foi categorizada a partir do ponto de corte estabelecido para a resposta à pergunta sobre o tempo transcorrido desde o último episódio de consumo e os usuá- 
rios não tinham a informação de como sua resposta seria analisada.

\section{Considerações Finais}

O conjunto das evidências aqui apresentadas indica que, para um efetivo tratamento e ocorrência mais frequente de cessação do uso de crack entre os usuários que chegam aos serviços de saúde, deve haver uma ampla investigação das condições do usuário, não limitada ao histórico e aos padrões de consumo do crack, mas incluindo, por exemplo, problemas relacionados à saúde sexual e reprodutiva, registro de estressores psicossociais, o envolvimento de familiares em situações de uso do crack e a possibilidade de sua inclusão nos atendimentos.

\section{Referências}

Albernaz, E. P., Menezes, A. M. B., César, J. A., Victora, C. G., Barros, F. C., \& Halpern, R. (2003). Fatores de risco associados à hospitalização por bronquiolite aguda no período pós-neonatal. Revista de Saúde Pública, 37, 48593. doi:10.1590/S0034-89102003000400014

American Psychological Association. (2002). DSM IV TR. Porto Alegre, RS: ArtMed.

Andretta, I., \& Oliveira, M. S. (2008). Efeitos da entrevista motivacional em adolescentes infratores. Estudos de Psicologia (Campinas), 25(1), 45-53. doi:10.1590/S0103-166X2008 000100005

Beck, A. T., Ward, C. H., Mendelson, M., Mock, J., \& Erbaugh, J. (1961). An inventory for measuring depression. Archives of General Psychiatry, 4(1), 561-571. doi:10.1001/archpsyc.1961.01710120031004

Battjes, R. J., Gordon, M. S., O'Grady, K. E., Kinlock, T. W., \& Carswell, M. A. (2003). Factors that predict adolescent motivation for substance abuse treatment. Journal of Substance Abuse Treatment, 24(3), 221-232. doi:10.1016/S07405472(03)00022-9

Bordin, S., Figlie, N. B., \& Laranjeira, R. (2004). Aconselhamento em dependência química: Cocaína e crack. São Paulo, SP: Roca.

Cahill, M., Adinoff, B., Hosig, H., Muller, K., \& Pulliam, C. (2003). Motivation for treatment preceding and following a substance abuse program. Addictive Behaviors, 28(1), 67-79. doi:10.1016/S0306-4603(01)00217-9

Carlini, E. A., Galduróz, J. C. F., Carlini, C. M., Silva, A. A. B., Noto, A. R. N., Fonseca, A. M., \& Sanchez, Z. M. (2006). II Levantamento Domiciliar sobre o Uso de Drogas Psicotrópicas no Brasil: Estudo envolvendo as 108 maiores cidades do país: 2005. São Paulo, SP: Centro Brasileiro de Informações Sobre Drogas Psicotrópicas. Recuperado em http://www.obid.senad.gov.br/portais/OBID/biblioteca/documentos/Publicacoes/ Sem_logo/329784.pdf

Carlini, E. A., Galduróz, J. C. F., Noto, A. R., \& Nappo, S. A. (2002). I Levantamento Domiciliar sobre o Uso de Drogas Psicotrópicas no Brasil: Estudo envolvendo as 107 maiores cidades do país: 2001. São Paulo, SP: Centro Brasileiro de Informações Sobre Drogas Psicotrópicas. Recuperado em http://abramd. org/wpcontent/uploads/2014/06/I_Levantamento_Domiciliar_sobre_o_Uso_de_Drogas_ Psicotr\%C3\%B3picas_no_Brasil.pdf

Chen, K., \& Kandel, D. B. (1998). Predictors of cessation of marijuana use: An event history analysis. Drug and Alcohol Dependence, 50(2), 109121. doi:10.1016/S0376-8716(98)00021-0

Cunha, J. A. (2001). Manual da versão em português das Escalas Beck. São Paulo, SP: Casa do Psicólogo.

Cunha, P. J., Nicastri, S., Gomes, L. P., Moino, R. M., \& Peluso, M. A. (2004). Alterações neuropsicológicas em dependentes de cocaína/crack internados: Dados preliminares. Revista Brasileira de Psiquiatria, 26(2), 103-106. doi:10.1590/ S1516-44462004000200007

Del Prette, Z. A. P., \& Del Prette, A. (2001). Inventário de Habilidades Sociais: Manual de aplicação e interpretação. São Paulo, SP: Casa do Psicólogo.

Duailibi, L. B., Ribeiro, M., \& Laranjeira, R. (2008). Profile of cocaine and crack users in Brazil. $\mathrm{Ca}$ dernos de Saúde Pública, 24(Supl. 4), 545-557. doi:10.1590/S0102-311X2008001600007

Farabee, D., McCann, M., Brecht, M. L., Cousins, S. J., Antonini, V. P., Lee, A. B., ...Rawson, R. A. (2013). An analysis of relapse prevention factors and their ability to predict sustained abstinence following treatment completion. The American Journal on Addictions, 22(3), 206-211. doi:10.1111/j.1521-0391.2012.00328.x 
Ferreira, O. F., Filho, Turchi, M. D., Laranjeira, R., \& Castelo, A. (2003). Perfil sociodemográfico e de padrões de uso entre dependentes de cocaína hospitalizados. Revista de Saúde Pública, 37(6), 751-759. doi:10.1590/S003489102003000600010

Ferreira, P. E. M., \& Martini, R. K. (2001). Cocaína: Lendas, história e abuso. Revista Brasileira de Psiquiatria, 23(2), 96-99. doi:10.1590/S151644462001000200008

García de Jesús, M. C., \& Ferriani, M. G. C. (2008). School as a "protective factor" against drugs: Perceptions of adolescents and teachers [Special issue]. Revista Latino-Americana de Enfermagem, 16, 590-594. doi:10.1590/S010411692008000700014

Ghitza, U. E., Preston, K. L., Epstein, D. H., Kuwabara, H., Endres, C. J., Bencherif, B., \& Gorelick, D. A. (2010). Brain mu-opioid receptor binding predicts treatment outcome in cocaine-abusing outpatients. Biological Psychiatry, 68(8), $697-$ 703. doi:10.1016/j.biopsych.2010.05.003

Gorelick, D. A., Kim, Y. K., Bencherif, B., Boyd, S. J., Nelson, R., Copersino, M., \& Frost, J. J. (2005). Imaging brain mu-opioid receptors in abstinent cocaine users: Time course and relation to cocaine craving. Biological Psychiatry, 57(12), 1573-1582. doi:10.1016/j.biopsych.2005.02.026

Guimarães, C. F., Santos, D. V. V., Freitas, R. C., \& Araujo, R. B. (2008). Perfil do usuário de crack e fatores relacionados à criminalidade em unidade de internação para desintoxicação no Hospital Psiquiátrico São Pedro de Porto Alegre (RS). Revista de Psiquiatria do Rio Grande do Sul, 30(2), 101-108. doi:10.1590/S010181082008000300005

Horta, R. L., Horta, B. L., \& Pinheiro, R. T. (2006). Drogas: Famílias que protegem e que expõem adolescentes ao risco. Jornal Brasileiro de Psiquiatria, 55(4), 268-272. doi:10.1590/ S0047-20852006000400002

Horta, R. L., Horta, B. L., Rosset, A. P., \& Horta, C. L. (2011). Perfil dos usuários de crack que buscam atendimento em Centros de Atenção Psicossocial. Cadernos de Saúde Pública, 27(11), $2263-$ 2270. doi:10.1590/S0102-311X2011001100019

Hughes, A., Sathe, N., \& Spagnola, K. (2009). State estimates of substance use from the 2006-2007 National Surveys on Drug Use and Health. Office of Applied Studies, Substance Abuse and
Mental Health Services Administration (NSDUH Series H-35, HHS Publication No. SMA 09-4362). Rockville, MD. Retrieved from http:// store.samhsa.gov/product/State-Estimates-of-Substance-Use-From-the-2006-2007-National-Surveys-on-Drug-Use-and-Health-NSDUH-/SMA09-4362

Karch, S. B. (1999). Cocaine: History, use, abuse. Journal of the Royal Society of Medicine, 92(8), 393-397. doi:10.1177/014107689909200803

Kessler, F., \& Pechansky, F. (2008). Uma visão psiquiátrica sobre o fenômeno do crack na atualidade. Revista de Psiquiatria do Rio Grande do Sul, 30(2), 96-98. doi:10.1590/S010181082008000300003

Laranjeira, R., Madruga, C. S., Pinsky, I., Caetano, R., Ribeiro M., \& Mitsuhiro, S. (2013). II Levantamento Nacional de Álcool e Drogas (LENAD II). São Paulo, SP: Instituto Nacional de Políticas Públicas do Álcool e Outras Drogas. Recuperado em http://inpad.org.br/lenad/resultados/cocaina-e-crack/resultados-preliminares/

Lopes, C. S., \& Coutinho, E. S. F. (1999). Transtornos mentais como fatores de risco para o desenvolvimento de abuso/dependência de cocaína: Estudo caso-controle. Revista de Saúde Pública, 33(5), 477-486. doi:10.1590/S003489101999000500007

Mehta, S. H., Sudarshi, D., Srikrishnan, A. K., Celentano, D. D., Vasudevan, C. K., Anand, S., \& Solomon, S. S. (2012). Factors associated with injection cessation, relapse and initiation in a community-based cohort of injection drug users in Chennai, India. Addiction, 107(2), 349-358. doi:10.1111/j.1360-0443.2011.03602.x

Narvaez, J. C. M., Jansen, K., Pinheiro, R. T., Kapczinski, F., Silva, R. A., Pechansky, F., \& Magalhães, P. V. (2014). Psychiatric and substance-use comorbidities associated with lifetime crack cocaine use in young adults in the general population. Comprehensive Psychiatry, 55(6), 1369-1376. doi:10.1016/j. comppsych.2014.04.02

Oliveira, L. G., \& Nappo, S. A. (2008). Caracterização da cultura de crack na cidade de São Paulo: Padrão de uso controlado. Revista de Saúde Pública, 42(4), 664-671. doi:10.1590/S003489102008005000039

Orsi, M. M., \& Oliveira, M. S. (2006). Avaliando a motivação para mudança em dependentes de co- 
caína. Estudos de Psicologia (Campinas), 23(1), 3-12. doi:10.1590/S0103-166X2006000100001

Pesce, R. P., Assis S. G., Avanci, J. Q., Santos, N.C., Malaquias. J. V., \& Carvalhaes, R. (2005). Adaptação transcultural, confiabilidade e validade da escala de resiliência. Cadernos de Saúde Pública, 21(2), 436-48. doi:10.1590/S0103166X2006000100001

Pratta, E. M. M., \& Santos, M. A. (2006). Reflexões sobre as relações entre drogadição, adolescência e família: Um estudo bibliográfico. Estudos de Psicologia (Natal), 11(3), 315-322. doi:10.1590/ S1413-294X2006000300009

Ramos, S. P. (2003). A psicanálise e os transtornos por uso de substâncias psicoativas (Tese de doutorado, Universidade Federal de São Paulo, Escola Paulista de Medicina, São Paulo, SP, Brasil).

Rodrigues, M. A. P., Facchini, L. A., \& Lima, M. S. (2006). Modificações nos padrões de consumo de psicofármacos em localidade do Sul do Brasil. Revista de Saúde Pública, 40(1), 107-114. doi:10.1590/S0034-89102006000100017

Rodrigues, V. S., Horta, R. L., Szupszynski, K. P. D. R., Souza, M. C., \& Oliveira, M. S. (2013). Revisão sistemática sobre tratamentos psicológicos para problemas relacionados ao crack. Jornal Brasileiro de Psiquiatria, 62(3), 208-216. doi:10.1590/S0047-20852013000300005

Sanchez, Z. M., Oliveira, L. G., Ribeiro, L. A., \& Nappo, S. A. (2010). O papel da informação como medida preventiva ao uso de drogas entre jovens em situação de risco. Ciência e Saúde Coletiva, 15(3), 699-708. doi:10.1590/S141381232010000300012
Schenker, M., \& Minayo, M. C. S. (2005). Fatores de risco e de proteção para o uso de drogas na adolescência. Ciência e Saúde Coletiva, 10(3), 707717. doi:10.1590/S1413-81232005000300027

Szupszynski, K. P. D. R., \& Oliveira, M. S. (2008). Adaptação brasileira da University of Rhode Island Change Assessment (URICA) para usuários de substâncias ilícitas. Psico-USF, 13(1), 31-39. doi:10.1590/S1413-82712008000100005

Vasconcelos, A. G. G., Almeida, R. M. V., Nobre, F. F. (2001). Path analysis and multi-criteria decision making: An approach for multivariate model selection and analysis in health. Annals of Epidemiology, 11(6), 377-384. doi:10.1016/ S1047-2797(01)00232-0

Victora, C. G., Huttly, S. R., Fuchs, S. C., \& Olinto, M. T. A. (1997). The role of conceptual frameworks in epidemiological analysis: A hierarchical approach. International Journal of Epidemiology, 26(1), 224-227. doi:10.1093/ije/26.1.224
Recebido: 15/01/2014

$1^{a}$ revisão: 08/08/2014

$2^{a}$ revisão: 06/10/2014

$3^{a}$ revisão: $21 / 10 / 2014$

$4^{a}$ revisão: $23 / 10 / 2014$

Aceite final: 24/10/2014 